\title{
Thrombotic Microangiopathy Score as a New Predictor for Neurologic Outcome in Patients Undergoing Targeted Temperature Management After Out-of-hospital Cardiac Arrest
}

\section{Taeyoung Kong}

Yonsei University College of Medicine

\section{Hye Sun Lee}

Yonsei University College of Medicine

\section{Soyoung Jeon}

Yonsei University College of Medicine Jong Wook Lee

Konyang University Hospital

\section{Hyun Soo Chung}

Yonsei University College of Medicine

\section{Sung Phil Chung}

Yonsei University College of Medicine

Je Sung You ( $\nabla$ youjsmd@yuhs.ac)

Yonsei University College of MedicineGangnam Severance Hospital https://orcid.org/0000-0002-20746745

\section{Original research}

Keywords: Out-of-hospital cardiac arrest, Targeted temperature management, Thrombotic microangiopathy score, Mortality, Predictor, Schistocytes

Posted Date: July 8th, 2020

DOI: https://doi.org/10.21203/rs.3.rs-40096/v1

License: @ (i) This work is licensed under a Creative Commons Attribution 4.0 International License. Read Full License 


\section{Abstract}

Background: Given the morphological characteristics of schistocytes, thrombotic microangiopathy (TMA) score can be beneficial as it can be quickly and serially measured without additional effort or costs. This study aimed to investigate whether the serial TMA scores until $48 \mathrm{~h}$ post admission are associated with clinical outcomes in patients undergoing targeted temperature management (TTM) after out-of-hospital cardiac arrest (OHCA).

Methods:We retrospectively evaluated a cohort of 185 patients using a prospective registry. We analyzed the TMA score at admission and after 12, 24, and 48 hours. The primary outcome measures were poor neurologic outcome at discharge and 30-day mortality.

Results:Increased TMA scores at all measured time points were independent predictors of poor neurologic outcomes and 30-day mortality, with the TMA score at time-12 showing the strongest correlation (OR, 3.008; $95 \% \mathrm{Cl}, 1.707-5.3 ; p=0.001$ and $\mathrm{HR}, 1.517 ; 95 \% \mathrm{Cl}, 1.196-1.925 ; p=0.001$.Specifically, TMA score $\geq 2$ at time-12 was closely associated with increased predictability of poor neurologic outcome (OR, $6.302 ; 95 \% \mathrm{Cl}$ : 2.841-13.976; $p<0.001)$ and 30-day mortality (HR, 2.656; 95\% Cl: 1.675-4.211; $p<0.001)$.

Conclusions: Increased TMA scores predicted the neurologic outcome and 30-day mortalityin patients undergoing TTM after OHCA. In addition to the benefit of being quickly and serially measured by using an automated hematology analyzer without additional effort or costs, this finding indicates that the TMA score may be a helpful tool for rapid risk stratification and identification of the need for intensive care in patients with ROSC after OHCA.

\section{Introduction}

Cardiac arrest (CA) causes approximately 500,000 deaths annually in the Europe and US [1]. Further, the survival rate after out-of-hospital cardiac arrest (OHCA) is less than $15 \%$ and remains poor [1]. OHCA also has high morbidity rate including neurologic disability [2]. Regardless of causes, CA and resuscitation lead to severe damages in multiple organs because of the associated hypoxemia, ischemia, and reperfusion [3], with the severity varying between patients. Accordingly, effective post-CA care requires identification and mitigation of the precipitating cause of CA and ischemia-reperfusion injury to multiple organ systems $[3,4]$. Recent guidelines have recommended targeted temperature management (TTM) as the neuroprotective intervention in resuscitation care of $\mathrm{CA}[3,5,6]$. Refractory shock inducing recurrent CA or multiorgan failure (MOF) typically results in deaths within the first 24 hours after return of spontaneous circulation (ROSC), while neurological injury causes later deaths after CA [7]. In the pathophysiology of multiorgan injury after $\mathrm{CA}$, cardiovascular ischemia/reperfusion injury and cardiovascular toxicity are significantly associated with excessive levels of inflammatory cytokine activation and catecholamines, among other contributing factors [7]. Systemic ischemia/reperfusion injury after ROSC leads to the release of inflammatory cytokines and the development of systemic inflammatory response syndrome that mimics sepsis without infection [7]. Clinically, post CA syndrome (PCAS), also known as sepsis-like syndrome, mimics the immunologic and coagulation disorders in patients with severe sepsis [8]. Despite advances in post-resuscitative care, a significant proportion of patients will have a poor neurological outcome and a high risk of mortality $[3,9]$. 
A multimodal approach is recommended to improve the accuracy of prognostication of CA survivors [3]. The application of new biomarkers that easily and quickly predict the development of unfavorable neurologic outcome and mortality after post-resuscitation may improve the prognosis of patients by enabling innovative monitoring, early aggressive treatment, and therapeutic strategies [10-12]. Thrombotic microangiopathy (TMA) is characterized by three important elements including higher levels of lactate dehydrogenase, thrombocytopenia, and fragmented erythrocytes (schistocytes) [13]. Several critical conditions lead to the development of systemic endothelial injury in critically ill patients $[14,15]$. In endothelial injury, severe hemolysis from mechanical damage in the circulation occasionally produce schistocytes in turbulent areas of the microcirculation that are partly occluded by platelet aggregations [14, 16]. The International Council for Standardization in Hematology (ICSH) Schistocyte Working Group recommended that the more than $1 \%$ schistocytes on a peripheral blood smear is an important criterion for identifying TMA [13]. Moreover, the occurrence of schistocytes in blood reflects a high risk of thrombocytopenia-associated multiple organ failure (TAMOF) [17]. Schistocytes indicate specific

characteristics of increased red cell distribution width (RDW) and hemoglobin distribution width (HDW), and microcytic hyperchromic red blood cells (RBCs) $[15,17]$. Based on the development and changes in the morphological characteristics of schistocytes, the TMA score was developed as a rapid, simple marker to screen for TAMOF in critically ill patients [15]. An automated complete blood cell (CBC) analyzer can automatically and easily determine the TMA score based on RBC parameters and the volume/hemoglobin concentration $(\mathrm{V} / \mathrm{HC})[15,17]$.

We previously found that increased TMA scores significantly predicted short-term mortality in patients with severe sepsis and septic shock $[15,18]$. To the best of our knowledge, no studies have reported that the new TMA score can predict the clinical outcomes of patients with OHCA. As such, we aimed to investigate whether the serial TMA scores over time were significantly associated with neurologic outcomes at hospital discharge and 30-day mortality in patients undergoing TTM after OHCA. Towards this goal, we investigated the association between the development and morphological changes of schistocytes and severity of organ damage in patients undergoing TTM after OHCA. We hypothesized that the TMA score could be used to predict the severity of organ damage in TTM patients who achieved sustained ROSC after OHCA.

\section{Methods}

\section{Study design and population}

This retrospective, observational cohort study was performed between July 2015 and May 2019 based on a prospective registry of the emergency department (ED) of Yonsei University College of Medicine affiliated with Severance Hospital, which is a tertiary-level referral hospital with a population of average 100,000 patients per year. The study was approved by the institutional review board (IRB) of Yonsei University Health System (3-2019-0308). Because of the retrospective nature of the study, the need for informed consent was waived by the IRB of Yonsei University Health System (3-2019-0308).

We included consecutive patients who were prospectively integrated into the Critical leaders, Optimal care Of Life threatening post cardiopulmonary resuscitation (CPR) (COOL) protocol as a critical pathway (CP) 
program. However, we retrospectively conducted data analysis based on OHCA records from the prospective registry of ED COOL CP. Considering the theoretical characteristics of TMA score, we excluded patients who received a transplant or prosthetic valves and those undergoing chemotherapy within 14 days from this study. To validate the usefulness of TMA scores over time, we also excluded patients who were transferred to our ED from another hospital, those who transferred out to another hospital within the first $72 \mathrm{~h}$, and those who died within 24 hours. Figure 1 summarizes the patient inclusion process.

\section{COOL protocol}

Since September 2011, we have implemented the COOL CP in our institutions as part of a quality improvement initiative to provide proper and professional treatment of TTM with bundle management to patients who had achieved sustained ROSC after OHCA. In the ED, emergency physicians screened candidates for the $\mathrm{COOL} \mathrm{CP}$ program as soon as feasible. According to the predetermined protocol based on the advanced cardiac life support guidelines published by the American Heart Association (AHA) [3], a multidisciplinary treatment group involving an cardiologist, intensivist, neurologist, and emergency physicians immediately identifies the patient status and simultaneously provide intensive and critical care. We provided post-CA care to patients who achieved sustained ROSC after OHCA following a standardized protocol within at least 72 hours after $\mathrm{CP}$ activation. The COOL CP program is available 24 hours a day, 7 days a week. According to this protocol, we actively applied TTM with a target core body temperature of $32{ }^{\circ} \mathrm{C}-36{ }^{\circ} \mathrm{C}$ for all adult non-traumatic survivors who were unable to obey commands. We strictly controlled blood pressure, glucose, electrolyte, ventilator minute volume, and fluid balance and performed essential emergency procedures. Patients were deemed ineligible for the CP based on the following criteria: age $<18$ years; pregnancy; trauma; intracranial hemorrhage; active gastrointestinal bleeding; terminal illness; do-notresuscitate status; and pre-existing coma prior to CA.

\section{Data collection}

Basic characteristics including age; sex; body mass index (BMI); and comorbidities including hypertension, diabetes mellitus, malignancy, and cerebrovascular, pulmonary, and prior arrhythmia, cardiac disease such as coronary artery disease, and cardiomyopathy were investigated. Data on CA characteristics including first monitored rhythm, presence of a witness on collapse, etiology of arrest (cardiac or non-cardiac origin), lowflow time (defined as the time with active CPR by a bystander and/or a medical provider), no-flow time (defined as time between the CA and initiation of CPR), and bystander CPR were also collected. The first available laboratory data for hematologic (white blood cell count) and chemistry (total bilirubin, lactate, total $\mathrm{CO}_{2}$, and glucose) parameters were also collected in patients who achieved sustained ROSC after OHCA.

\section{Assessment of TMA score}

The TMA score ranges from 0 to 5 points, with 1 point given for each of the following: (1) RDW $>15 \%$, (2) $\mathrm{HDW}>3.2 \%$, (3) percentage of microcytes $\geq 0.4 \%$ (percentage of microcytes indicates the percentage of RBCs smaller than $60 \mathrm{fL}$ ), (4) percentage of hyperchromic red cells $\geq 1.9 \%$ (percentage of hyperchromic red cells indicates the percentage of RBCs with more than $41 \mathrm{~g} / \mathrm{dL}$ of hemoglobin), and (5) platelet count $<140$ $\times 10^{9} / \mathrm{L}[15,17]$. All RBC parameters as well as the TMA score were measured using an automated blood cell 
analyzer (ADVIA 120; Siemens, Forchheim, Germany) $[15,17]$ and reported in the electronic health records. The percentages of microcytes and hyperchromic red cells were calculated using the V/HC cytogram [15, 17]. We analyzed the TMA scores from the time of ED admission to 48 hours. Venous blood was collected in ethylenediaminetetraacetic-containing vacutainers on ED admission (time-0) and 12 hours (time-12), 24 hours (time-24), and 48 hours (time-48) after admission.

\section{Statistical analysis}

The primary outcome measure was unfavorable neurologic outcome at hospital discharge, which was evaluated according to the cerebral performance category (CPC) scale $[19,20]$. The CPC scale score ranges from 1 to 5 points, with 1-2 defined as good outcome and 3-5 as bad outcome $[19,20]$. The secondary outcome measure was the 30-day all-cause mortality. The CPC score was determined by an investigator blinded to the study hypothesis through a review of the medical records. Clinicodemographic data were presented as medians and interquartile ranges (IQRs), means and standard deviations, percentages, or frequencies, as appropriate [19]. Continuous variables were compared using the two-sample t-test or MannWhitney U-test, and categorical variables using the $\chi^{2}$ test or Fisher's exact test $[15,19]$. To identify the significance of the differences between the groups over time, we conducted a linear two-factor mixed model using a repeated-measures covariance pattern and unstructured covariance within patients [15]. We identified two fixed effects for this model: the time effect (TMA from time- 0 to time-48) and the diagnostic effect for the clinical endpoint (good and bad neurologic outcome; survival and nonsurvival after 30 days) [15]. We performed univariable analyses to identify significant associations between the clinicodemographic data and study outcomes $[15,19]$. To identify independent predictors of 30 -day mortality and neurologic outcome during TTM after OHCA, variables with $\mathrm{p}<0.05$ in univariable analysis were entered into multivariable logistic regression analysis and multivariable Cox regression analysis, respectively [19].

The predictive performance of the TMA score was evaluated according to the receiver-operating characteristic (ROC) curves and the area under the curve (AUC) [15]. The optimal cutoff of the TMA score to discriminate between good and bad neurologic outcome was determined used Youden's method. The results were presented as odds ratios (ORs) and 95\% confidential intervals (Cls) [15]. The net reclassification improvement (NRI) and integrated discrimination improvement (IDI) values were measured to verify how the addition of the TMA score improved the prediction model for clinical outcomes [21]. To determine the statistical significance of the IDI and NRI indices, we resampled 1,000 times using a standard bootstrap method [21, 22]. The 30-day survival was plotted using Kaplan-Meier curves and compared using log-rank test [19]. Unlike previous studies that estimated the cut-off values based only on events, we estimated these values using the technique devised by Contal and O'Quigley to determine the optimal cut-off values for the dichotomization of the clinical outcome variable for 30-day mortality based on time-to-event data [19]. The optimal cut-off points were selected by maximizing the HR [19, 23]. All statistical analyses were performed using SAS version 9.2 (SAS Institute Inc., Cary, NC) and MedCalc Statistical Software version 16.4.3 (MedCalc Software bvba, Ostend, Belgium), and $p<0.05$ was considered significant.

\section{Results}




\section{Study population, clinical evaluation, and treatment}

A total of 224 adult CA patients who achieved ROSC were registered COOL CP program during the study period. Of them, 39 patients were excluded, leaving 185 patients in the final analysis (Fig. 1). In total, 69 and 116 patients belonged to the good outcome and the bad outcome group, respectively. The incidence of allcause 30 -day mortality was $43.2 \%$ and unfavorable outcome was $62.7 \%$. Table 1 shows the patients' clinical characteristics stratified by neurologic outcomes and mortality. The mean age of the overall population was $60.1 \pm 16.4$ years, and 131 (70.8\%) of them were men. Among the patients who achieved ROSC after OHCA, nonsurvivors were older than survivors. Patients with bad neurologic outcome were also older than those with good outcomes. The incidence of diabetes was higher in patients with poor neurologic outcome and in the nonsurvivors. They were also more likely to have a non-shockable rhythm, a non-cardiac etiology, a longer no-flow time, and a longer low-flow time. Table 1 also shows the distributions of the first available laboratory results after ROSC. Patients who died within 30 days or those with poor neurologic outcome had significantly higher TMA scores than those who did not. As shown in Fig. 2, there were significant differences between groups in the TMA scores according to the 30-day mortality (group, $p<0.001$; time, $p<$ 0.001 ; and group $\times$ time, $p=0.139$ ) and neurologic outcome after ED admission (group, $p<0.001$; time, $p<$ 0.001 ; and group $\times$ time, $p=0.241)($ Fig. $2 A, B)$. 
Table 1

Clinical characteristics of the patients stratified by 30-day mortality and neurologic outcome

\begin{tabular}{|c|c|c|c|c|c|c|c|}
\hline \multirow{2}{*}{$\begin{array}{l}\text { Variables } \\
\text { Survival }\end{array}$} & \multirow{2}{*}{$\begin{array}{l}\text { Total } \\
\text { Death }\end{array}$} & \multicolumn{3}{|c|}{ 30-day Mortality } & \multicolumn{3}{|c|}{ Neurologic outcome } \\
\hline & & \multirow[t]{2}{*}{$\mathrm{P}$} & Good & Poor & \multirow[t]{2}{*}{$P$} & & \\
\hline$N=185(100 \%)$ & $\begin{array}{l}N=105 \\
(56.8 \%)\end{array}$ & & $\begin{array}{l}N=80 \\
(43.2 \%)\end{array}$ & $\begin{array}{l}N=69 \\
(37.3 \%)\end{array}$ & & \multicolumn{2}{|l|}{$\begin{array}{l}N=116 \\
(62.7 \%)\end{array}$} \\
\hline Age (years) & $\begin{array}{l}60.05 \pm \\
16.43\end{array}$ & $\begin{array}{l}56.25 \pm \\
16.50\end{array}$ & $\begin{array}{l}65.05 \pm \\
15.04\end{array}$ & $<0.001 *$ & $\begin{array}{l}54.36 \pm \\
15.42\end{array}$ & $\begin{array}{l}63.44 \pm \\
16.14\end{array}$ & $<.001 *$ \\
\hline Male sex [n (\%)] & $131(70.81)$ & $80(76.19)$ & $51(63.75)$ & 0.065 & $96(73.28)$ & $35(26.71)$ & 0.422 \\
\hline $\mathrm{BMI}\left(\mathrm{kg} / \mathrm{m}^{2}\right)$ & $\begin{array}{l}22.74 \pm \\
4.00\end{array}$ & $\begin{array}{l}22.37 \pm \\
3.86\end{array}$ & $\begin{array}{l}23.27 \pm \\
4.16\end{array}$ & 0.137 & $\begin{array}{l}22.86 \pm \\
3.77\end{array}$ & $\begin{array}{l}22.67 \pm \\
4.15\end{array}$ & 0.752 \\
\hline \multicolumn{8}{|l|}{$\begin{array}{l}\text { Medical history, } \\
\text { [n (\%)] }\end{array}$} \\
\hline Hypertension & $72(38.92)$ & $38(36.19)$ & $34(42.50)$ & 0.383 & $21(30.43)$ & $51(43.97)$ & 0.068 \\
\hline $\begin{array}{l}\text { Diabetes } \\
\text { mellitus }\end{array}$ & $47(25.41)$ & 19(18.10) & $28(35.00)$ & $0.009 *$ & $9(13.04)$ & $38(32.76)$ & $0.003^{*}$ \\
\hline $\begin{array}{l}\text { Chronic } \\
\text { pulmonary } \\
\text { disease }\end{array}$ & $12(6.49)$ & $4(3.81)$ & $8(10.00)$ & 0.09 & $1(1.45)$ & $11(9.48)$ & $0.034 *$ \\
\hline $\begin{array}{l}\text { Cardiovascular } \\
\text { disease }\end{array}$ & $42(22.70)$ & $27(25.71)$ & $15(18.75)$ & 0.263 & 18(26.09) & $24(20.69)$ & 0.397 \\
\hline Arrhythmia & 13(7.03) & $8(7.62)$ & $5(6.25)$ & 0.718 & $5(7.25)$ & $8(6.90)$ & ${ }_{0.999}$ \\
\hline $\begin{array}{l}\text { Cerebrovascular } \\
\text { disease }\end{array}$ & $11(5.95)$ & $5(4.76)$ & $6(7.50)$ & 0.535 & $2(2.90)$ & $9(7.76)$ & 0.215 \\
\hline Malignancy & $16(8.65)$ & $9(8.57)$ & $7(8.75)$ & 0.966 & $6(8.70)$ & $10(8.62)$ & 0.986 \\
\hline \multicolumn{8}{|l|}{$\begin{array}{l}\text { Cardiac arrest } \\
\text { characteristics }\end{array}$} \\
\hline $\begin{array}{l}\text { Witnessed } \\
\text { collapse, [n (\%)] }\end{array}$ & & & & $0.004^{*}$ & & & 0.064 \\
\hline No & $75(40.54)$ & $33(31.43)$ & $42(52.50)$ & & $22(31.88)$ & $53(45.69)$ & \\
\hline Yes & $110(59.46)$ & $72(68.57)$ & $38(47.50)$ & & $47(68.12)$ & $63(54.31)$ & \\
\hline $\begin{array}{l}\text { Bystander CPR, } \\
\text { [n (\%)] }\end{array}$ & & & & $0.017^{*}$ & & & 0.103 \\
\hline No & $59(31.89)$ & $26(24.76)$ & $33(41.25)$ & & $17(24.64)$ & $42(36.21)$ & \\
\hline Yes & $126(68.11)$ & $79(75.24)$ & $47(58.75)$ & & $52(75.36)$ & 74(63.79) & \\
\hline
\end{tabular}




\begin{tabular}{|c|c|c|c|c|c|c|c|}
\hline \multirow{2}{*}{$\begin{array}{l}\text { Variables } \\
\text { First monitored } \\
\text { rhythm, [n (\%)] }\end{array}$} & \multirow[t]{2}{*}{ Total } & \multicolumn{2}{|c|}{ 30-day Mortality } & \multicolumn{4}{|c|}{ Neurologic outcome } \\
\hline & & & & $\hat{0}_{0.001 *}$ & & & $<.001 *$ \\
\hline Non-shockable & $109(58.92)$ & $43(40.95)$ & $66(82.50)$ & & 18(26.09) & $91(78.45)$ & \\
\hline Shockable & $76(41.08)$ & $62(59.05)$ & $14(17.50)$ & & $51(73.91)$ & $25(21.55)$ & \\
\hline $\begin{array}{l}\text { Etiology of } \\
\text { arrest, [n (\%)] }\end{array}$ & & & & $<.001 *$ & & & $<.001 *$ \\
\hline Non-cardiac & $66(35.68)$ & $25(23.81)$ & $41(51.25)$ & & $7(10.14)$ & $59(50.86)$ & \\
\hline Cardiac & $119(64.32)$ & $80(76.19)$ & $39(48.75)$ & & $62(89.86)$ & $57(49.14)$ & \\
\hline $\begin{array}{l}\text { No-flow time } \\
\text { (min) }\end{array}$ & $2.10 \pm 4.44$ & $\begin{array}{l}1.40 \pm \\
3.20\end{array}$ & $\begin{array}{l}3.01 \pm \\
5.56\end{array}$ & $0.022^{*}$ & $\begin{array}{l}1.22 \pm \\
2.78\end{array}$ & $\begin{array}{l}2.62 \pm \\
5.12\end{array}$ & $0.017 *$ \\
\hline $\begin{array}{l}\text { Low-flow time } \\
\text { (min) }\end{array}$ & $\begin{array}{l}24.73 \pm \\
15.73\end{array}$ & $\begin{array}{l}20.09 \pm \\
12.99\end{array}$ & $\begin{array}{l}30.83 \pm \\
16.95\end{array}$ & $<.001 *$ & $\begin{array}{l}16.62 \pm \\
11.49\end{array}$ & $\begin{array}{l}29.55 \pm \\
15.96\end{array}$ & $<.001 *$ \\
\hline \multicolumn{8}{|l|}{$\begin{array}{l}\text { Laboratory data } \\
\text { after ROSC }\end{array}$} \\
\hline $\begin{array}{l}\text { White blood cell } \\
\text { count }\left(10^{\wedge} 3 \mu \mathrm{L}\right)\end{array}$ & $\begin{array}{l}12.66 \pm \\
5.79\end{array}$ & $\begin{array}{l}12.71 \pm \\
5.37\end{array}$ & $\begin{array}{l}12.59 \pm \\
6.32\end{array}$ & 0.897 & $\begin{array}{l}13.62 \pm \\
5.48\end{array}$ & $\begin{array}{l}12.09 \pm \\
5.91\end{array}$ & 0.081 \\
\hline $\begin{array}{l}\text { Total bilirubin } \\
(\mathrm{mg} / \mathrm{dL})\end{array}$ & $0.59 \pm 0.50$ & $\begin{array}{l}0.56 \pm \\
0.38\end{array}$ & $\begin{array}{l}0.64 \pm \\
0.63\end{array}$ & 0.316 & $\begin{array}{l}0.55 \pm \\
0.29\end{array}$ & $\begin{array}{l}0.62 \pm \\
0.59\end{array}$ & 0.275 \\
\hline $\begin{array}{l}\text { Lactate } \\
(\mathrm{mmol} / \mathrm{L})\end{array}$ & $\begin{array}{l}10.56 \pm \\
4.45\end{array}$ & $\begin{array}{l}9.20 \pm \\
4.33\end{array}$ & $\begin{array}{l}12.39 \pm \\
3.96\end{array}$ & $<0.001 *$ & $\begin{array}{l}8.65 \pm \\
4.18\end{array}$ & $\begin{array}{l}11.72 \pm \\
4.22\end{array}$ & $<0.001 *$ \\
\hline tCO2 (mmol/L) & $\begin{array}{l}17.48 \pm \\
5.32\end{array}$ & $\begin{array}{l}17.22 \pm \\
4.81\end{array}$ & $\begin{array}{l}17.81 \pm \\
5.95\end{array}$ & 0.469 & $\begin{array}{l}16.28 \pm \\
4.49\end{array}$ & $\begin{array}{l}18.19 \pm \\
5.66\end{array}$ & $0.013^{*}$ \\
\hline $\begin{array}{l}\text { Glucose } \\
\text { (mg/dL) }\end{array}$ & $\begin{array}{l}279.8 \pm \\
120.2\end{array}$ & $\begin{array}{l}259.3 \pm \\
104.3\end{array}$ & $\begin{array}{l}306.7 \pm \\
134.3\end{array}$ & $0.01^{*}$ & $\begin{array}{l}260.7 \pm \\
99.1\end{array}$ & $\begin{array}{l}291.2 \pm \\
130.2\end{array}$ & 0.075 \\
\hline $\begin{array}{l}\text { TMA score } \\
\text { Time-0 (point) }\end{array}$ & $0.72 \pm 0.89$ & $\begin{array}{l}0.55 \pm \\
0.82\end{array}$ & $\begin{array}{l}0.95 \pm \\
0.94\end{array}$ & $0.003^{*}$ & $\begin{array}{l}0.39 \pm \\
0.62\end{array}$ & $\begin{array}{l}0.92 \pm \\
0.97\end{array}$ & $<.001 *$ \\
\hline $\begin{array}{l}\text { TMA score } \\
\text { Time-12 (point) }\end{array}$ & $1.13 \pm 1.09$ & $\begin{array}{l}0.85 \pm \\
0.93\end{array}$ & $\begin{array}{l}1.53 \pm \\
1.17\end{array}$ & $<.001 *$ & $\begin{array}{l}0.64 \pm \\
0.79\end{array}$ & $\begin{array}{l}1.45 \pm \\
1.14\end{array}$ & $<.001 *$ \\
\hline $\begin{array}{l}\text { TMA score } \\
\text { Time-24 (point) }\end{array}$ & $1.34 \pm 1.25$ & $\begin{array}{l}1.08 \pm \\
1.17\end{array}$ & $\begin{array}{l}1.78 \pm \\
1.26\end{array}$ & $<0.001 *$ & $\begin{array}{l}0.86 \pm \\
0.96\end{array}$ & $\begin{array}{l}1.69 \pm \\
1.32\end{array}$ & $<.001 *$ \\
\hline $\begin{array}{l}\text { TMA score } \\
\text { Time-48 (point) }\end{array}$ & $1.48 \pm 1.23$ & $\begin{array}{l}1.17 \pm \\
1.06\end{array}$ & $\begin{array}{l}2.07 \pm \\
1.32\end{array}$ & $<.001 *$ & $\begin{array}{l}0.96 \pm \\
0.96\end{array}$ & $\begin{array}{l}1.90 \pm \\
1.27\end{array}$ & $<.001 *$ \\
\hline
\end{tabular}

\section{TMA score as a predictor of poor neurologic outcome for patients undergoing TTM after OHCA}


In univariable analysis, the TMA scores at several time points revealed significant differences in neurologic outcomes of patients undergoing TTM after OHCA (Supplementary Table S1). In further multivariable logistic regression analyses, elevated value of TMA score was independent in prediction of unfavorable neurologic outcomes at time-0 $(\mathrm{OR}, 2.27 ; 95 \% \mathrm{Cl}, 1.242-4.151 ; \mathrm{p}=0.008)$, time-12 (OR, 3.008; 95\% Cl, 1.7075.3; $p=0.001)$, and time-24 (OR, 1.797; 95\% Cl, 1.178-2.74; $p=0.007)$ (Table 2). The AUC for the TMA score on ED admission for predicting bad neurologic outcome was $0.645(\mathrm{P}<0.001)$ (Fig. $2 \mathrm{C})$. The ROC curve analysis of the prediction of bad neurologic outcome indicated that the highest AUCs for the TMA score was 0.704 (IQR, 0.632-0.777; p < 0.001) at time-12 (Fig. 2D). Accordingly, the optimal cut-off value of the TMA score at time-0 was at time-12 was 2 (sensitivity, 48.6 [IQR, 38.8-58.5]; specificity: 87.0 [IQR, 76.7-93.9]) (Fig. 2D). A TMA score $\geq 2$ at time-12 was significantly associated with increased predictability of unfavorable neurologic outcome (OR, 6.302; 95\% Cl: 2.841-13.976; $\mathrm{p}<0.001)$ (Fig. 2D).

\section{TMA score as a predictor of 30-day mortality for patients undergoing TTM after OHCA}

The univariable analysis also showed significant differences in the TMA scores at different time points between survivors and nonsurvivors within 30-days (Supplementary Table S2). Further multivariable Cox regression analyses demonstrated that increased TMA score at time- $0(\mathrm{HR}, 1.334 ; 95 \% \mathrm{Cl}, 1.032-1.724 ; \mathrm{p}=$ $0.028)$, time-12 (HR, 1.517; $95 \% \mathrm{Cl}, 1.196-1.925 ; \mathrm{p}=0.001)$, and time-48 $(\mathrm{HR}, 1.452 ; 95 \% \mathrm{Cl}, 1.114-1.892 ; \mathrm{p}$ $=0.006$ ) (Table 3) were independent predictors of 30-day mortality The optimal cut-off value of the TMA score at time-12 was 2 . Considering time to event, TMA $\geq 2$ at time- 12 was significantly associated with increased 30-day mortality (Fig. 3A, B). TMA score $\geq 2$ at time-12 was associated with increased predictability of 30-day mortality in patients undergoing TTM after OHCA (HR, 2.656; 95\% Cl: 1.675-4.211; $\mathrm{p}$ $<0.001$ ) (Fig. 3A, B).

Predictive power when TMA was included as a conventional risk factor for patients undergoing TTM after OHCA

As statistical indication for verifying the enhancement of reclassification in a nested model, many studies proposed net reclassification improvement (NRI) and integrated discrimination improvement (IDI), thus indicating how the addition of a new factor to traditional risk factors improves the prediction model for clinical outcomes. At time-12 after admission, the current study demonstrated that the combination of TMA with traditional factors produced significantly positive NRI and IDI for the prediction of poor neurologic outcomes in patients undergoing TTM after OHCA. The combination of TMA at time-12 with conventional risk factors also revealed statistically significant improvement in IDI for predicting short-term mortality at 30 days (Table 4). 
Table 4

Comparison of the predictive performance for poor neurologic outcome between the model with and without the TMA score according to integrated discrimination improvement (IDI) and continuous net reclassification improvement (NRI)

\begin{tabular}{|c|c|c|c|c|}
\hline Prediction model & IDI (95\% Cl) & $P$ & $\begin{array}{l}\text { NRI } \\
(95 \% \mathrm{Cl})\end{array}$ & $P$ \\
\hline Null model & Reference & & Reference & \\
\hline Null model + TMA score Time-0 & $0.055(-0.054-0.164)$ & 0.321 & $0.033(0.006-0.059)$ & $0.016^{\star}$ \\
\hline Null model + TMA score Time-12 & $0.148(0.015-0.28)$ & $0.029 *$ & $0.083(0.042-0.125)$ & $<0.001^{\star}$ \\
\hline Null model + TMA score Time-24 & $0.086(-0.014-0.186)$ & 0.092 & $0.045(0.015-0.075)$ & $0.003^{*}$ \\
\hline Null model + TMA score Time-48 & $0.115(-0.001-0.232)$ & 0.053 & $0.053(0.022-0.085)$ & $0.001^{\star}$ \\
\hline \multicolumn{5}{|c|}{$\begin{array}{l}\text { *P }<0.05 \text {; OR, odds ratio; } \mathrm{Cl} \text {, confidence interval; SOFA, sequential organ failure assessment; TMA, } \\
\text { thrombotic microangiopathy. }\end{array}$} \\
\hline
\end{tabular}

Table 2. Multivariable logistic regression analysis for predictors of unfavorable neurologic outcome.

\begin{tabular}{|c|c|c|c|c|c|c|c|c|}
\hline \multirow{2}{*}{ Varialle } & \multicolumn{8}{|c|}{ Mutiverinle lgistic regression } \\
\hline & $\operatorname{or}(95 \% \mathrm{C})$ & $\boldsymbol{P}$ & $\operatorname{Or}(95 \% \mathrm{CI})$ & $\boldsymbol{P}$ & $\operatorname{OR}(95 \% \mathrm{CI})$ & $\boldsymbol{P}$ & $\operatorname{ares} \% \mathrm{Cl}$ & $\boldsymbol{P}$ \\
\hline Age & $1.025(0994-1057)$ & 0.111 & 1.024(0991-1059) & 0.153 & $1.018(0984-1053)$ & 0295 & 1_012(0978-1048) & $0 \_487$ \\
\hline Witnessed collepse & $0570(0.196-1654)$ & 0301 & $0.402(0.127-1272)$ & 0121 & $0.621(0.215-1.794)$ & 0379 & $0.622(0.205-1887)$ & 0.401 \\
\hline Bystander CPR & $1048(0250-4397)$ & 0949 & $1365(0304-6119)$ & 0.685 & $0.963(0.228-4.162)$ & 0959 & $1309(0287-5979)$ & 0729 \\
\hline hitial shockable thythm & $0329(0.124-0.877)$ & $0.026^{*}$ & $0.258(0.094-0.706)$ & 0.0108* & $0327(0.120-0.888)$ & $0.028 *$ & $0307(0.109-0862)$ & $0.025^{*}$ \\
\hline No fowtime & $1.026(0.880-1.197)$ & 0.742 & $1.037(0.895-1202)$ & 0.628 & $1.034(0.887-1205)$ & 0669 & $1.057(0902-1238)$ & 0.495 \\
\hline Low flow time & $1.074(1.034-1.115)$ & $<0001^{*}$ & 1_.075(1_033-1118) & $<0001^{*}$ & 1_073(1_031-1.116) & $<0001^{*}$ & 1_081(1_.037-1_126) & $<0001^{*}$ \\
\hline \multicolumn{9}{|l|}{ Fiology of amest } \\
\hline Non-Cardiar origin & Reference & & Reference & & Reference & & Reference & \\
\hline Cardiar origin & $0.243(0.074-0.794)$ & 0_019* & $0.168(0.046-0.617)$ & 0.60\%* & $0.180(0.052-0.627)$ & $0.007 *$ & $0.208(0.056-0.767)$ & $0.018 *$ \\
\hline Fistroy of diabetes & $2.167(0.678-6930)$ & 0.192 & $1.870(0-547-6389)$ & 0318 & $2251(0.689-7357)$ & 0.179 & 2441(0_699-8517) & 0.162 \\
\hline Arterial tCO2 & $1111(0.997-1237)$ & 0.056 & 1.086(0.970-1215) & 0.152 & $1.103(0.985-1236)$ & 0.09 & 1.094(0.974-1228) & 0.129 \\
\hline Arterial batate & 1.135(1.014-1271) & $0.027^{*}$ & 1.1668(0949-1202) & 0273 & $1.079(0.959-1214)$ & 0208 & 1.028(0.910-1.161) & 0659 \\
\hline TMA sxere Time-0 & $2270(1242-4151)$ & O_LIB: & & & & & & \\
\hline TMA sxere Time-12 & & & 3.008(1.707-5300) & $<0001^{*}$ & & & & \\
\hline TMA scere Time-24 & & & & & $1.797(1.178-2740)$ & 0_ant* & & \\
\hline TMA sxare Time 48 & & & & & & & $1.889(1213-2941)$ & 0_olos* \\
\hline
\end{tabular}

*P<0.05; OR, odds ratio; $\mathrm{CI}$, confidence interval;CPR, cardiopulmonary resuscitation; $\mathrm{tCO} 2$, total carbon dioxide; TMA, thrombotic microangiopathy.

Table 3. Multivariable Cox proportional hazard regression analysis for predictors of 30-day mortality. 


\begin{tabular}{|c|c|c|c|c|c|c|c|c|}
\hline \multirow{2}{*}{ Varialle } & \multicolumn{8}{|c|}{ Muthorible Cox prepertinal haxard regressio } \\
\hline & HIR $(65 \%$ G) & $\boldsymbol{P}$ & HIR $(95 \%$ Ch) & $\boldsymbol{P}$ & IR $(95 \% \mathrm{Cl}$ & $\boldsymbol{P}$ & IR e5\% CI & $\boldsymbol{P}$ \\
\hline Age & $1029\left(1 \_011-1 \_047\right)$ & 0001* & 1031(1_011-1_050) & $0002 *$ & 1_021(1_01-1_(441) & $0.039 *$ & $1.022(1001-1.043)$ & 0_0138* \\
\hline Witnessed collapse & $0575(0341-0971)$ & $0038 *$ & $0545(03140944)$ & $0.031 *$ & $0.599(0334-1074)$ & 0.1885 & $0.529(0283-0985)$ & $0.045^{*}$ \\
\hline Bystander CPR & $0827(0.391-1.747)$ & 0.618 & $0950(0.434-2080)$ & 0.899 & $0.655(0.287-1.496)$ & 0315 & $0.767(0315-1866)$ & 0558 \\
\hline Initial shockeble mythm & 0554(0281-1_(090) & 0.087 & $0520(0266-1.016)$ & 0.056 & $0.540(0259-1.124)$ & 0.10 & $0.574(0255-1289)$ & 0179 \\
\hline No fowtime & 1016(0946-1_091) & 0.668 & 1032(0.957-1_114) & 0.411 & 1_026(0951-1_107) & 05018 & $1.052(0969-1.142)$ & 0224 \\
\hline Low flow time & $1023(1.018-1.039)$ & $00013 *$ & $1020(1$ (104-1_036) & $0012 *$ & 1.024(1.016-1_(142) & 0_on $7^{*}$ & 1_026(1008-1 (045) & 0_oto \\
\hline \multicolumn{9}{|l|}{ Fiology of arrest } \\
\hline Non-Candiar origin & Reference & & Reference & & Reference & & Reference & \\
\hline Cardiar origin & $0853(0.498-1 / 461)$ & 0.563 & $0815(0.466-1.424)$ & 0.472 & $0.724(0396-1321)$ & 0292 & $0.639(0337-1213)$ & 0171 \\
\hline Histroy of diabetes & 1314(0.751-2300) & 0.339 & $0974(0.537-1.767)$ & 0.931 & $1308(0.685-2.500)$ & 0.416 & $0.918(0.445-1.893)$ & 0816 \\
\hline Arterial batate & 1.128(1_054-1208) & $<0.01 *$ & $1099(1.024-1.178)$ & $0000^{*}$ & 1.079(0999-1_166) & 0.553 & $1.053(0967-1146)$ & 0234 \\
\hline TMA scere Timeo & 1334(1_032-1_724) & $0.028 *$ & & & & & & \\
\hline TMA scere Time-12 & & & $1517(1.196-1925)$ & <0_0.01* & & & & \\
\hline TMA sxere Time-24 & & & & & $1249(1001-1565)$ & 0.049* & & \\
\hline TMA sxere Time 48 & & & & & & & $1.452(1.114-1892)$ & 0_1016* \\
\hline
\end{tabular}

$* \mathrm{P}<0.05$; HR, hazard ratio; CI, confidence interval;CPR, cardiopulmonary resuscitation; TMA, thrombotic microangiopathy.

\section{Discussion}

The current study demonstrated that higher TMA scores were associated with mortality after 30 days and poor neurologic outcome in patients undergoing TTM after OHCA. Increased TMA score was an independent predictor of 30-day mortality and unfavorable neurologic outcomes. This was consistent with a previous study for severe sepsis and septic shock in which sepsis patients who died 30 days after ED admission had significantly higher TMA scores than those who survived, with increased TMA scores being significant predictors of 30-day mortality [15]. Clinically, sepsis is characterized by a systemic inflammatory response accompanying an infection [10,24]. In contrast, the ROSC after CA induces systemic ischemic reperfusion injury and consequently leads to sterile inflammatory damages after ischemic/reperfusion injury in the entire body without infection $[3,25]$.

It is difficult to completely understand the exact pathophysiologic processes underlying the clinical relationship between TMA score and poor outcomes after OHCA. However, several hypotheses could be proposed in this study. First, PCAS can be a contributing factor to the high mortality rate and severe brain damage of patients who achieve ROSC after CA [2,3]. The systemic and sterile inflammation is the most important component of PCAS [8, 25]. Second, whole-body ischemia/reperfusion induced by endothelial injury contributes to thrombotic occlusion of the vessels following activation of coagulation and impairment of fibrinolysis $[26,27]$. Several studies have demonstrated that aggregation, deformability, and the shape of RBC are altered in critical illness and different diseases [28]. 
Given the development and changes in the morphological characteristics of schistocytes, the TMA score was developed to screen for TAMOF in critically ill patients as a rapid, simple marker [15]. With respect to erythrocytes, the hemorheological state after ischemia and reperfusion may negatively impact the deformability and aggregation behavior of RBCs through several complex process including acute phase reactions, free radical and inflammatory response, hemodynamic deteriorations, and coagulopathy [29]. Yu et al assessed the rheological properties of RBC after ischemic/reperfusion and reported that stiffer erythrocytes resulting from reduced deformability help increase vascular resistance by limiting movement to small tissue capillaries. This eventually leads to no-reflow phenomenon from the cells being trapped in capillaries [30]. Accordingly, the unfavorable neurologic outcome is attributed to the cerebral microvascular occlusion that causes the no-reflow phenomenon [31]. Many animal and clinical studies demonstrated a more severe degree of microvascular changes is associated with poor outcomes including severe hypoxic brain injury, immediate death without ROSC, and high mortality [32].

In patients who achieve ROSC after CA, PCAS mimics the immunologic and coagulation disorders in severe sepsis [8]. the development of disseminated intravascular coagulation (DIC) as a serious disease results in an increased tendency of bleeding, microvascular thrombosis, thrombocytopenia, and multiple organ dysfunction, consequently affecting the clinically unfavorable outcomes of patients with PCAS [32]. DIC and TMA show similar laboratory findings and presenting symptoms [32]. Although microvascular thrombosis is mainly caused by platelet activation in both DIC and TMA, DIC is significantly associated with all coagulation and fibrinolysis systems, while TMA is significantly associated with vascular endothelial cells [33]. Thrombotic thrombocytopenic purpura and hemolytic uremic syndrome are known as the classic forms of TMA [34]. Despite differences in the mechanisms of underlying DIC and TMA [33], several different conditions, such as atypical hemolytic uremic syndrome, DIC, and drug toxicities, can be also considered as different forms of TMA [34]. It is difficult to use the schistocyte count as a complete indicator for the initial diagnosis and confirmation of TMA and DIC [13]. Various conditions including severe infection, pregnancy, and leukemia frequently produce schistocytes $[13,35]$. Nevertheless, the International Council for Standardization in Hematology (ICSH) guideline states that presence of $>1 \%$ schistocytes without other moderate changes in RBCs upon a peripheral blood smear can be considered as a critical criterion for the diagnosis of TMA [13].

Although changes in the microcirculation are known to play an important role in the severity of organ damage after CA, only few studies have investigated the characteristic changes for RBC deformability after $\mathrm{CA}$ and ischemic/reperfusion injury [36]. To investigate rheological changes of RBCs after ischemicreperfusion injury, Lee et al. evaluated the RBC deformability by three-dimensional shapes of RBCs after asphyxial CA in rats using three-dimensional laser interferometric microscopy. They did not find changes in three-dimensional shapes and cell deformability in RBCs during CPR and in 60 min after ROSC [36]. Nemeth et al. demonstrated that the erythrocyte deformability significantly deteriorate 1-3 days after limb ischemic/reperfusion [29]. The limited number of studies prompts us to refer to the significant association between sepsis and DIC. The increased development of schistocyte may be closely associated with the severity of sepsis and septic shock. Moreover, the increased rigidity and decreased deformability of RBCs during sepsis are clinically relevant for sepsis patients as they significantly influence outcomes [37]. Specifically, this progressive decrease of RBC deformability, which occurs over the next 2 to 8 days since the 
onset of infections, is closely associated with multiple organ dysfunction [37, 38]. Given that PCAS has similar clinical characteristics to severe sepsis with respect to systemic and sterile inflammation, the increase in the number and changes in the characteristics of schistocytes may also be similar to severe sepsis and septic shock $[8,25]$. Unlike the study by Lee et al. that simply evaluated changes in threedimensional shapes and cell deformability in RBCs during CPR and in 60 min after ROSC [36], we used the TMA score, which represents changes in the morphological characteristics of schistocytes, to predict 30-day mortality and unfavorable neurologic outcomes for patients undergoing TTM after OHCA. To the best of our knowledge, this is the first study to report increased TMA score, which reflects significant changes in the morphological characteristics of schistocytes, within 12-24 h after ED admission. Importantly, we found that increased TMA score is a reliable predictor of clinical outcomes in patients undergoing TTM after OHCA.

Ko et al. reported that the optimal cutoff values of TMA scores at time- 0 and time-24 were 2 and 3 in patients with sepsis and TMA scores $\geq 2$ at time- 0 and $\geq 3$ at time-24 increased the predictability of 30-day mortality [15]. Similarly, in the present study, a TMA score $\geq 2$ at time-12 was closely associated with the increased predictability of 30-day mortality and unfavorable neurologic outcome in patients undergoing TTM after OHCA. Therefore, microvascular dysregulation and mechanical damage by RBC injury in ischemic reperfusion injury can play an important role in the formation of schistocytes [37]. Further prospective, multicenter trials are required to validate the prognostic value of cell deformability in RBCs in $12 \mathrm{~h}$ and $24 \mathrm{~h}$ after admission in patients with PCAS undergoing TTM after OHCA.

Some studies suggested that the automated counting of RBC fragments is helpful as a routine screening tool, with good agreement with microscopy and other several advantages $[13,35]$. The TMA score is a simple and rapidly measurable marker in clinical practice [15]. In our study, the specific automated hematology analyzer can automatically measure TMA score during a CBC count and report the TMA score without additional costs, time, or laboratory tests $[17,18]$. During the early stage of TTM after OHCA, the TMA score can be valuable because it can indicate the changes in the morphological characteristics of schistocytes rather than the absolute schistocyte counts [39]. Our findings support that the TMA score can improve the predictability for the prognosis and risk stratification of patients undergoing TTM after OHCA. This is in line with the findings of another study that the TMA score can be a useful ancillary marker during clinical decision in the early stage TTM after OHCA [39].

The current study has several limitations. First, the possibility of selection bias cannot be eliminated. Although we used a prospective $\mathrm{CP}$ with a predetermined standardized protocol, we retrospectively analyzed this using a cohort derived from the CP of a single center. Second, we could not directly compare between TMA scores from an automated hematology analyzer and the schistocyte count determined manually through a microscope. As we stated in our previous study, the TMA score was created based on significant changes in the morphological characteristics of schistocytes and is determined automatically. Finally, we could not investigate the association between ADAMTS-13 activity and TMA score. The present study evaluated the usefulness of the TMA score for predicting the severity of organ failure in patients undergoing TTM after OHCA. ADAMTS-13 activity is a critical factor in the pathophysiology of the classic form of TMA. Although we used a prospective registry of the CP, we could not measure ADAMTS-13 activity because it was 
not a mandatory biomarker in this registry. Further prospective, multicenter trials are required to validate the usefulness of the TMA scores as a prognostic factor in patients undergoing TTM after OHCA.

\section{Conclusions}

Increased TMA scores predicted the neurologic outcome and 30-day mortality in patients undergoing TTM after OHCA. In addition to the benefit of being quickly and serially measured by using an automated hematology analyzer without additional effort or costs, this finding indicates that the TMA score may be a helpful tool for rapid risk stratification and identification of the need for intensive care in patients with ROSC after OHCA.

\section{Abbreviations}

TMA

thrombotic microangiopathy; TTM:targeted temperature management; OHCA:out-of-hospital cardiac arrest; CA:Cardiac arrest; MOF:multiorgan failure; ROSC:return of spontaneous circulation; PCAS:post cardiac arrest syndrome; ICSH:International Council for Standardization in Hematology; TAMOF:thrombocytopeniaassociated multiple organ failure; RDW:red cell distribution width; HDW:hemoglobin distribution width; RBC:blood cell; CBC:complete blood cell; V/HC:volume/hemoglobin concentration; ED:emergency department; IRB:institutional review board; CPR:cardiopulmonary resuscitation COOL:Critical leaders, Optimal care Of Life threatening post CPR; CP:critical pathway; AHA:American Heart Association; BMI:body mass index; CPC:cerebral performance category:ROC:receiver-operating characteristic; AUC:area under the curve; ORs:odds ratios; Cis:95\% confidential intervals; NRI:net reclassification improvement; IDI; integrated discrimination improvement (IDI); DIC:disseminated intravascular coagulation

\section{Declarations}

\section{Acknowledgements}

None

\section{Authors' contributions}

TK, JWL, and JSY conceived and planned the study and were mainly responsible for its design. TK, and JSY collected data. TK, SK, HSL, SJ, and JSY were mainly responsible for analysing the data. TK and JSY wrote the first draft of the manuscript. All authors (TK, HSL, SJ, SK, JSY, HSC, JWL, and SPC) contributed to the interpretation of the finding and reviewed the manuscript. SK, HSL, SJ, SPC, and HSC reviewed the statistical analyses and made changes to the content of the manuscript. All authors authors(TK, HSL, SJ, SK, JSY, HSC, JWL, and SPC) have also provided intellectual contribution to the manuscript.

\section{Funding}

J.S.Y. received funding from the Basic Science Research Program of the National Research Foundation of Korea (NRF) funded by the Ministry of Science and ICT (NRF- 2018R1C1B6006159) and a faculty research 
grant from Yonsei University College of Medicine for 2019 (6-2019-0188).

\section{Availability of data and materials}

The dataset used and analyzed during the current study are available fromthe corresponding author on reasonable request.

\section{Ethics approval and consent to participate}

The study was approved by the institutional review board (IRB) of Yonsei University Health System (32019-0308). Because of the retrospective nature of the study, the need for informed consent was waived by the IRB of Yonsei University Health System (3-2019-0308).

\section{Consent for publication}

Not applicable.

\section{Competing interests}

J.S.Y. also received research funding from Siemens Health Care (3-2015-0140). However, the funds did not exceed $\$ 10,000$ per year. The founding sponsors had no role in the design of the study; in the collection, analyses, or interpretation of data; in the writing of the manuscript, and in the decision to publish the results. The other authors declare no conflict of interest.

\section{Authors' contributions}

TK, JWL, and JSY conceived and planned the study and were mainly responsible for its design. TK, and JSY collected data. TK, SK, HSL, SJ, and JSY were mainly responsible for analysing the data. TK and JSY wrote the first draft of the manuscript. All authors (TK, HSL, SJ, SK, JSY, HSC, JWL, and SPC) contributed to the interpretation of the finding and reviewed the manuscript. SK, HSL, SJ, SPC, and HSC reviewed the statistical analyses and made changes to the content of the manuscript. All authors authors(TK, HSL, SJ, SK, JSY, HSC, JWL, and SPC) have also provided intellectual contribution to the manuscript.

\section{References}

1. Girotra S, Chan PS, Bradley SM. Post-resuscitation care following out-of-hospital and in-hospital cardiac arrest. Heart. 2015;101(24):1943-9.

2. Benjamin EJ, Muntner P, Alonso A, Bittencourt MS, Callaway CW, Carson AP, et al. Heart Disease and Stroke Statistics-2019 Update: A Report From the American Heart Association. Circulation. 2019;139(10):e56-28.

3. Callaway CW, Donnino MW, Fink EL, Geocadin RG, Golan E, Kern KB, et al. Part 8: Post-Cardiac Arrest Care: 2015 American Heart Association Guidelines Update for Cardiopulmonary Resuscitation and Emergency Cardiovascular Care. Circulation. 2015;132(18 Suppl 2):465-82. 
4. Ahn C, Kim W, Cho Y, Choi KS, Jang BH, Lim TH. Efficacy of extracorporeal cardiopulmonary resuscitation compared to conventional cardiopulmonary resuscitation for adult cardiac arrest patients: a systematic review and meta-analysis. Sci Rep. 2016;6:34208.

5. Dankiewicz J, Cronberg T, Lilja G, Jakobsen JC, Belohlavek J, Callaway C, et al. Targeted hypothermia versus targeted Normothermia after out-of-hospital cardiac arrest (TTM2): A randomized clinical trialRationale and design. Am Heart J. 2019;217:23-31.

6. Du L, Ge B, Ma Q, Yang J, Chen F, Mi Y, et al. Changes in cardiac arrest patients' temperature management after the publication of 2015 AHA guidelines for resuscitation in China. Sci Rep. 2017;7(1):16087.

7. Jentzer JC, Chonde MD, Dezfulian C. Myocardial Dysfunction and Shock after Cardiac Arrest. Biomed Res Int. 2015;2015:314796.

8. Adrie C, Laurent I, Monchi M, Cariou A, Dhainaou JF, Spaulding C. Postresuscitation disease after cardiac arrest: a sepsis-like syndrome? Curr Opin Crit Care. 2004;10(3):208-12.

9. Ryoo SM, Lee DH, Lee BK, Youn CS, Kim YJ, Kim SJ, et al. Prognostic Factors for Re-Arrest with Shockable Rhythm during Target Temperature Management in Out-Of-Hospital Shockable Cardiac Arrest Patients. J Clin Med. 2019;8(9).

10. Kim JH, Park YS, Yoon CY, Lee HS, Kim S, Lee JW, et al. Delta Neutrophil Index for the Prediction of the Development of Sepsis-Induced Acute Kidney Injury in the Emergency Department. Shock. 2019. doi:10.1097/shk.0000000000001299.

11. Kellum JA, Prowle JR. Paradigms of acute kidney injury in the intensive care setting. Nat Rev Nephrol. 2018;14(4):217-30.

12. Tverring J, Vaara ST, Fisher J, Poukkanen M, Pettila V, Linder A. Heparin-binding protein (HBP) improves prediction of sepsis-related acute kidney injury. Ann Intensive Care. 2017;7(1):105.

13. Schapkaitz E, Mezgebe MH. The Clinical Significance of Schistocytes: A Prospective Evaluation of the International Council for Standardization in Hematology Schistocyte Guidelines. Turk J Haematol. 2017;34(1):59-63.

14. Saigo K, Jiang M, Tanaka C, Fujimoto K, Kobayashi A, Nozu K, et al. Usefulness of automatic detection of fragmented red cells using a hematology analyzer for diagnosis of thrombotic microangiopathy. Clin Lab Haematol. 2002;24(6):347-51.

15. Ko DR, Kong T, Lee HS, Kim S, Lee JW, Chung HS, et al. Usefulness of the Thrombotic Microangiopathy Score as a Promising Prognostic Marker of Septic Shock for Patients in the Emergency Department. J Clin Med. 2019;8(6).

16. Moake JL. Thrombotic microangiopathies. N Engl J Med. 2002;347(8):589-600.

17. Yoo JH, Lee J, Roh KH, Kim HO, Song JW, Choi JR, et al. Rapid identification of thrombocytopeniaassociated multiple organ failure using red blood cell parameters and a volume/hemoglobin concentration cytogram. Yonsei Med J. 2011;52(5):845-50.

18. Goag EK, Lee JW, Roh YH, Leem AY, Kim SY, Song JH, et al. A Simplified Mortality Score Using Delta Neutrophil Index and the Thrombotic Microangiopathy Score for Prognostication in Critically III Patients. Shock. 2018;49(1):39-43. 
19. Yune HY, Chung SP, Park YS, Chung HS, Lee HS, Lee JW, et al. Delta neutrophil index as a promising prognostic marker in out of hospital cardiac arrest. PLoS One. 2015;10(3):e0120677.

20. Chung SP, Yune HY, Park YS, You JS, Hong JH, Kong T, et al. Usefulness of mean platelet volume as a marker for clinical outcomes after out-of-hospital cardiac arrest: a retrospective cohort study. J Thromb Haemost. 2016;14(10):2036-44.

21. Kong T, Kim TH, Park YS, Chung SP, Lee HS, Hong JH, et al. Usefulness of the delta neutrophil index to predict 30-day mortality in patients with ST segment elevation myocardial infarction. Sci Rep. 2017;7(1):15718.

22. Kim J, Song TJ, Song D, Yoo J, Baek JH, Lee HS, et al. Prognostic value of urine dipstick proteinuria on mortality after acute ischemic stroke. Atherosclerosis. 2016;253:118-23.

23. Grigoriu BD, Scherpereel A, Devos P, Chahine B, Letourneux M, Lebailly P, et al. Utility of osteopontin and serum mesothelin in malignant pleural mesothelioma diagnosis and prognosis assessment. Clin Cancer Res. 2007;13(10):2928-35.

24. Umbro I, Gentile G, Tinti F, Muiesan P, Mitterhofer AP. Recent advances in pathophysiology and biomarkers of sepsis-induced acute kidney injury. J Infect. 2016;72(2):131-42.

25. Adrie C, Adib-Conquy M, Laurent I, Monchi M, Vinsonneau C, Fitting C, et al. Successful cardiopulmonary resuscitation after cardiac arrest as a "sepsis-like" syndrome. Circulation. 2002;106(5):562-8.

26. Ono Y, Hayakawa M, Maekawa K, Kodate A, Sadamoto Y, Tominaga N, et al. Fibrin/fibrinogen degradation products (FDP) at hospital admission predict neurological outcomes in out-of-hospital cardiac arrest patients. Resuscitation. 2017;111:62-7.

27. Adrie C, Monchi M, Laurent I, Um S, Yan SB, Thuong M, et al. Coagulopathy after successful cardiopulmonary resuscitation following cardiac arrest: implication of the protein $\mathrm{C}$ anticoagulant pathway. J Am Coll Cardiol. 2005;46(1):21-8.

28. Reggiori G, Occhipinti G, De Gasperi A, Vincent JL, Piagnerelli M. Early alterations of red blood cell rheology in critically ill patients. Crit Care Med. 2009;37(12):3041-6.

29. Nemeth N, Furka I, Miko I. Hemorheological changes in ischemia-reperfusion: an overview on our experimental surgical data. Clin Hemorheol Microcirc. 2014;57(3):215-25.

30. Yu SJ, Buerck JP, O'Rear EA, Whitsett TL. Possible erythrocyte contributions to and exacerbation of the post-thrombolytic no-reflow phenomenon. Biorheology. 2018;54(2-4):81-93.

31. Wada T, Gando S, Mizugaki A, Yanagida Y, Jesmin S, Yokota H, et al. Coagulofibrinolytic changes in patients with disseminated intravascular coagulation associated with post-cardiac arrest syndromefibrinolytic shutdown and insufficient activation of fibrinolysis lead to organ dysfunction. Thromb Res. 2013;132(1):e64-9.

32. Gando S, Wada T. Disseminated intravascular coagulation in cardiac arrest and resuscitation. J Thromb Haemost. 2019;17(8):1205-16.

33. Wada H, Matsumoto T, Suzuki K, Imai H, Katayama N, Iba T, et al. Differences and similarities between disseminated intravascular coagulation and thrombotic microangiopathy. Thromb J. 2018;16:14.

34. Benz K, Amann K. Thrombotic microangiopathy: new insights. Curr Opin Nephrol Hypertens. 2010;19(3):242-7. 
35. Zini G, d'Onofrio G, Briggs C, Erber W, Jou JM, Lee SH, et al. ICSH recommendations for identification, diagnostic value, and quantitation of schistocytes. Int J Lab Hematol. 2012;34(2):107-16.

36. Lee HJ, Lee S, Park H, Park Y, Shin J. Three-Dimensional Shapes and Cell Deformability of Rat Red Blood Cells during and after Asphyxial Cardiac Arrest. Emerg Med Int. 2019;2019:6027236.

37. Bateman RM, Sharpe MD, Singer M, Ellis CG. The Effect of Sepsis on the Erythrocyte. Int J Mol Sci. 2017;18(9).

38. Langenfeld JE, Livingston DH, Machiedo GW. Red cell deformability is an early indicator of infection. Surgery. 1991;110(2):398-403. discussion - 4.

39. Bellomo R, Martensson J, Eastwood GM. Metabolic and electrolyte disturbance after cardiac arrest: How to deal with it. Best Pract Res Clin Anaesthesiol. 2015;29(4):471-84.

\section{Figures}




\section{Patients enrolled COOL CP \\ (2015.7.1-2019.5.31) \\ $\mathrm{N}=224$}

- Exclusion ( $\mathrm{N}=39)$

Death within 24 hours $=12$

Transfer out within 72 hours $=4$

Prosthetic valve $=6$

Transplantation $=2$

Chemotherapy within 14 days $=4$

$\mathrm{DNAR}=11$

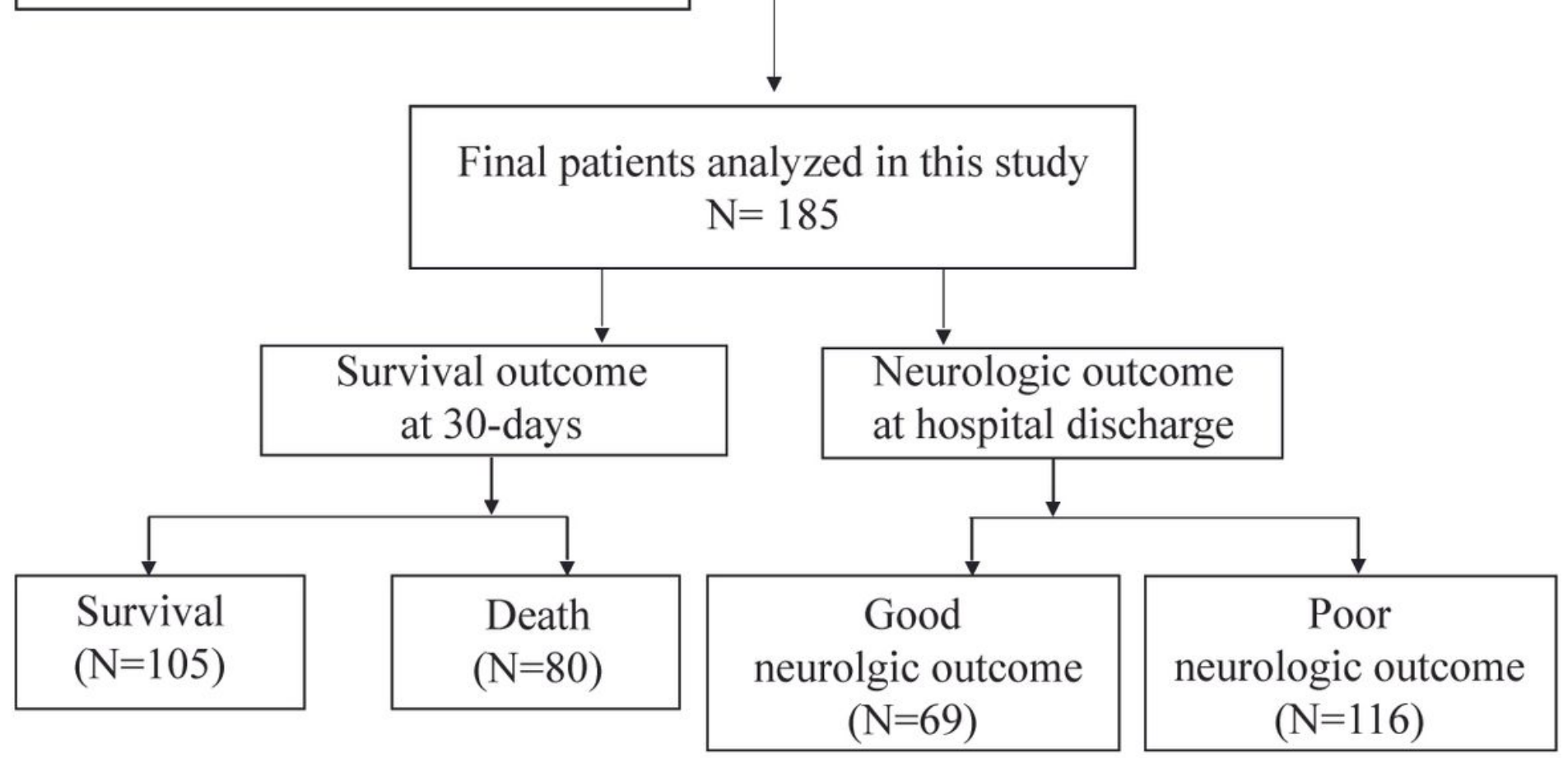

Figure 1

Flow diagram of patient inclusion. 
(A)

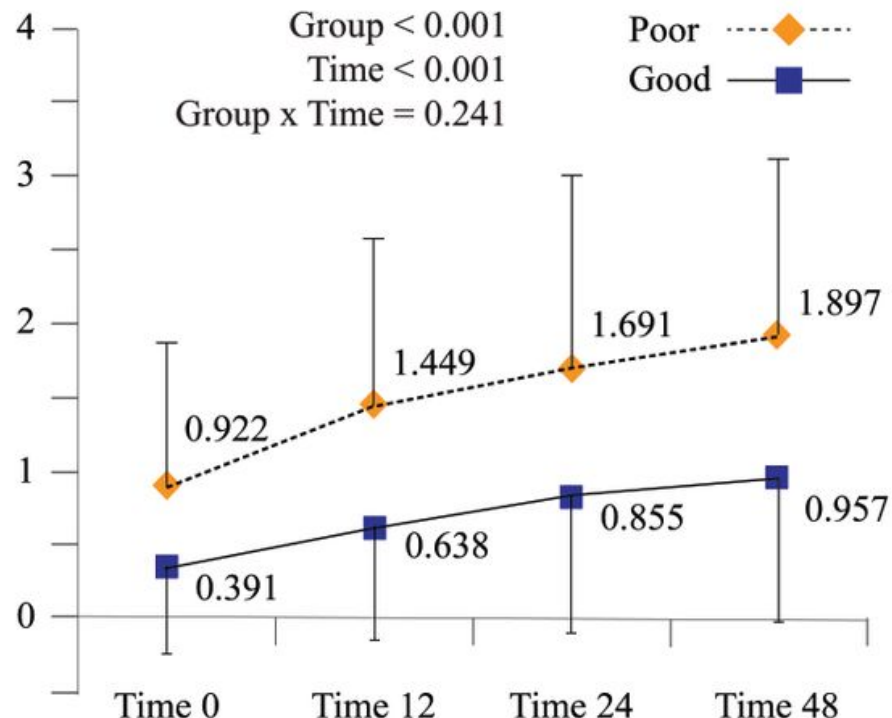

(C)

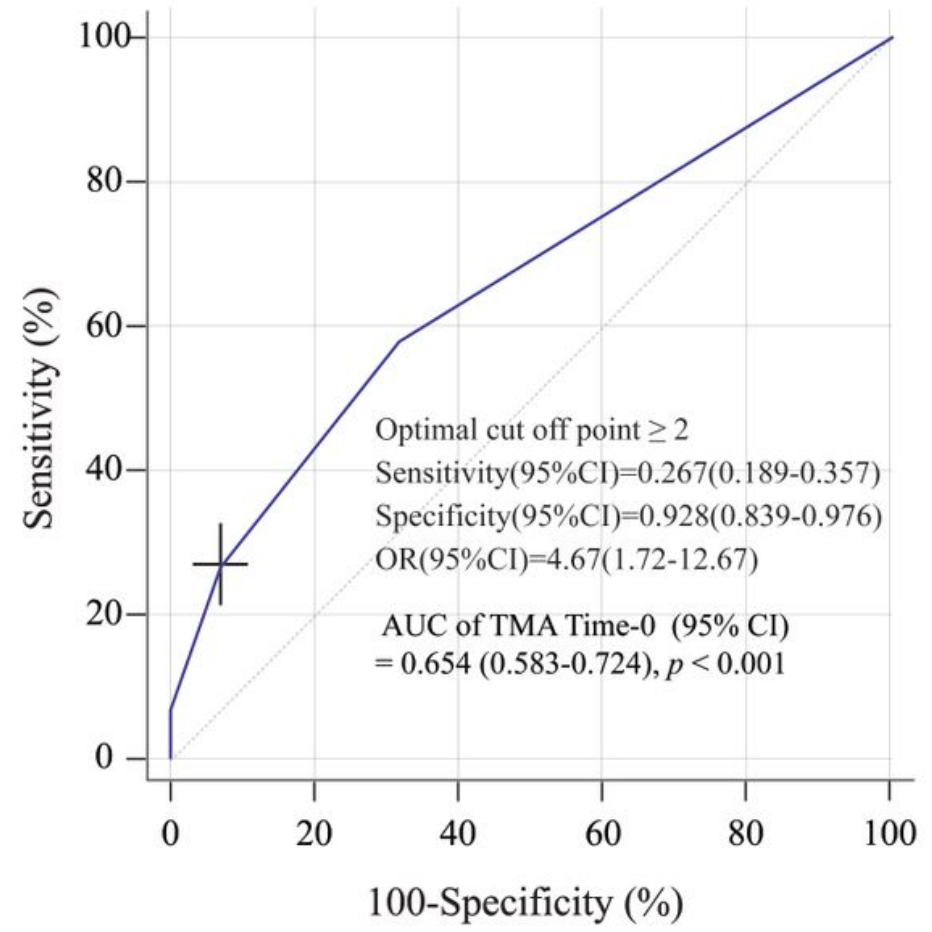

(B)

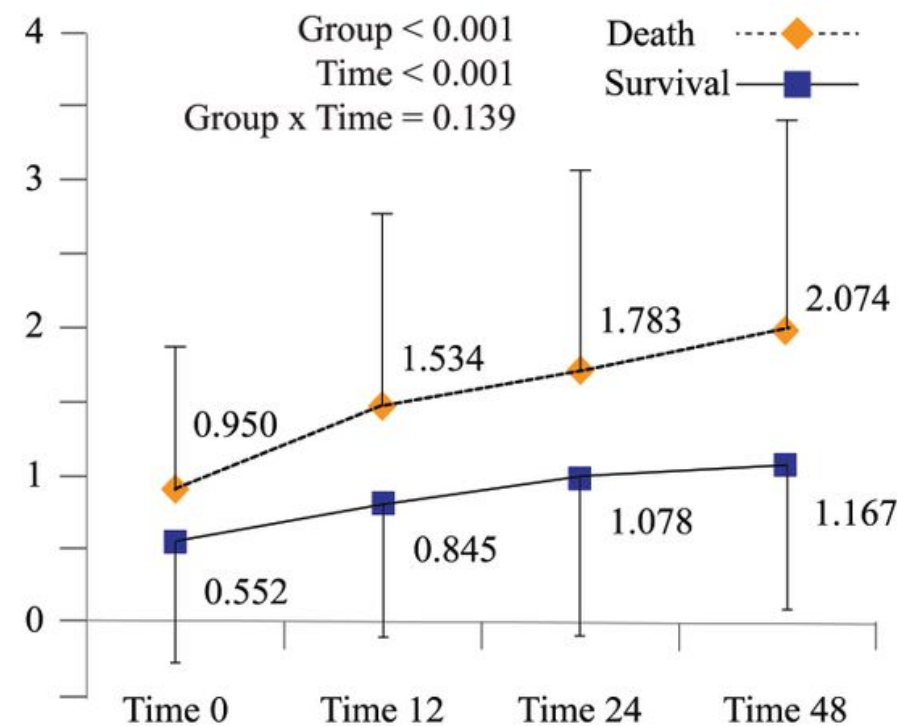

(D)

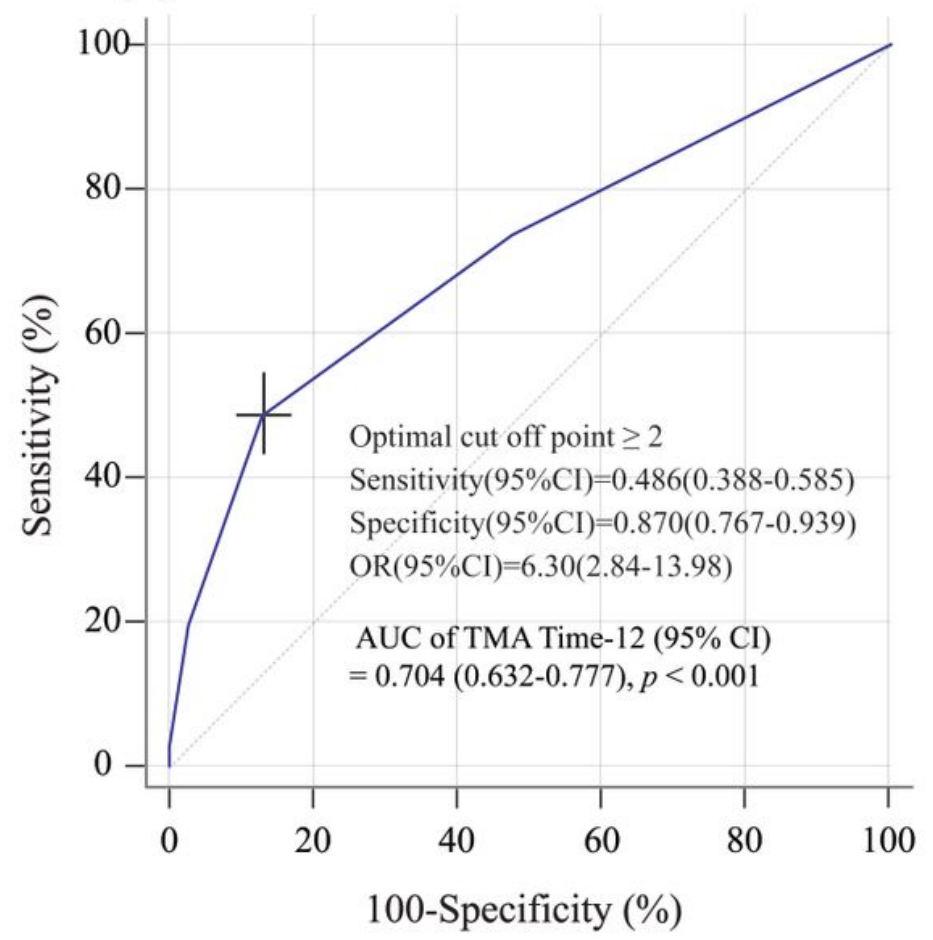

\section{Figure 2}

Linear mixed model of the TMA score to estimate significant differences between groups over time according to neurologic outcome (A) and 30-day mortality (B). The receiver operating characteristic curves for predictive capability of the TMA score at admission (C) and $12 \mathrm{~h}$ after admission (D) according to unfavorable neurologic outcome. 

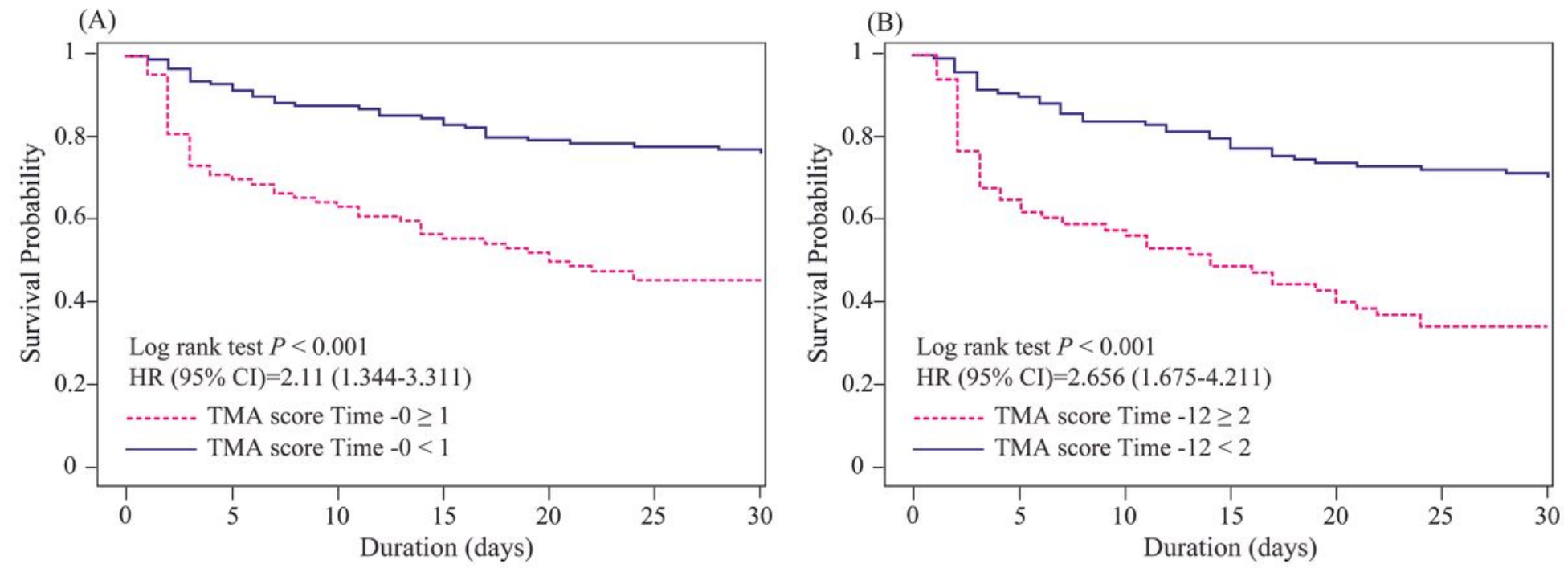

Figure 3

TMA score as a predictor of 30-day mortality. Higher TMA score at admission (A) and $12 \mathrm{~h}$ after admission (B) were significantly associated with an increased risk of 30-day mortality among patients with ROSC undergoing TTM after OHCA

\section{Supplementary Files}

This is a list of supplementary files associated with this preprint. Click to download.

- finalsubmissionSJTEOHCATMAsupplements.docx 